\title{
Low-Complexity and Distributed Energy Minimization in Multi-hop Wireless Networks
}

\author{
Longbi Lin, Xiaojun Lin, and Ness B. Shroff \\ Center for Wireless Systems and Applications (CWSA) \\ School of Electrical and Computer Engineering, Purdue University \\ West Lafayette, IN 47907, USA \\ \{llin, linx, shroff $\} @$ ecn.purdue.edu
}

\begin{abstract}
In this work, we study the problem of minimizing the total power consumption in a multi-hop wireless network subject to a given offered load. It is well-known that the total power consumption of multi-hop wireless networks can be substantially reduced by jointly optimizing power control, link scheduling, and routing. However, the known optimal crosslayer solution to this problem is centralized, and with high computational complexity. In this paper, we develop a lowcomplexity and distributed algorithm that is provably powerefficient. In particular, under the node exclusive interference model, we can show that the total power consumption of our algorithm is at most twice as large as the power consumption of the optimal (but centralized and complex) algorithm. Our algorithm is not only the first such distributed solution with provable performance bound, but its power-efficiency ratio is also tighter than that of another sub-optimal centralized algorithm in the literature.
\end{abstract}

Index Terms-Energy Aware Routing, Duality, Mathematical Programming/Optimization, Cross-Layer Optimization, Simulations

\section{INTRODUCTION}

There has been significant recent interest in developing control protocols for multi-hop wireless networks. Many applications can benefit from the deployment of these networks. For instance, sensors can form multi-hop wireless networks for such applications as habitat monitoring [1], and the management of sewer overflow events [2]. Vehicles can form multihop wireless networks to exchange safety messages and traffic information [3]. Wireless LAN devices can form multi-hop mesh networks to provide wireless broadband access [4].

A key issue in developing control protocols for multihop wireless networks is to reduce the energy or power consumption. This is obviously an important issue for batterypowered networks since the power consumption often limits the lifetime of the network. Even for networks with access to power sources, the transmission power of the communication links may still need to be properly controlled, e.g., due to health or regulatory concerns.

In this work, we are interested in the problem of minimizing the total power consumption of a multi-hop wireless network, subject to a given offered load. It is well-known that the total power consumption of multi-hop wireless networks can be substantially reduced by jointly optimizing power control, link scheduling, and routing. However, known optimal solutions require centralized computation and high computational complexity. In this paper, we propose a new low-complexity and distributed solution to this problem under a widely-used interference model, called the node-exclusive interference model. Using this model, the work in [5] developed a centralized solution that yielded a 3-approximation ratio (the resultant power consumption is within a factor of 3 from the optimal power consumption). In contrast, in this paper, we will obtain a $(2+\varepsilon)$-approximation algorithm that is fully distributed, where $\varepsilon>0$ is an arbitrarily small constant. To the best of our knowledge, our proposed algorithm is the first distributed solution in the literature with a provable performance bound.

Our solution approach is inspired by the recent progress in using imperfect scheduling algorithms to develop distributed cross-layer congestion control and scheduling algorithms in multi-hop wireless networks. We first formulate the energy minimization problem into a special form that naturally leads to a distributed solution. We then map the solution to corresponding components of the cross-layer control protocols, and rigorously establish the power-efficiency of the resulting algorithm.

Our work is also related to the study of energy-aware routing protocols for minimizing energy consumption and extending network lifetime [6]-[10]. These works assume that the system capacity is battery-limited instead of interference-limited and therefore do not consider scheduling constraints. In contrast, our work explicitly considers scheduling, jointly with power control and routing.

The intellectual contribution of this work is summarized as follows:

- We develop a low-complexity and distributed joint routing, power control, and scheduling algorithm for multihop wireless networks with provable power-efficiency ratio. Further, our algorithm can guarantee a better powerefficiency level than some existing centralized algorithms.

- Our solution cannot be obtained by extending the known optimal solution in the literature [11], [12]. Instead, we develop an optimization approach to the energy minimization problem that naturally leads to distributed solutions. We also develop rigorous techniques to identify the 
convergence properties and quantify the power-efficiency of the resulted control protocol.

The rest of this paper is organized as follows: in Section II, we present the system model and formulate the energy minimization problem. In Section III, we present our algorithm and discuss its implications. In Section IV, we discuss our main result on the convergence properties and the power-efficiency of the dual algorithm. Numerical results are provided in Section V. Concluding remarks are presented in Section VI.

\section{Problem Formulation}

We model a wireless multi-hop network by a directed graph $G(V, E)$, where $V$ is the set of vertices representing the nodes, and $E$ is the set of edges representing the communication links. We use $N_{o}(v)$ and $N_{i}(v)$ to denote the sets of outgoing and incoming links of node $v$, respectively. Their union $N(v)$ forms the set of all links incident on node $v$.

The system is time-slotted. We adopt the following nodeexclusive interference model that is used to characterize $\mathrm{FH}$ CDMA and UWB system with perfect orthogonal spreading codes and low power-spectrum density. Under this model, a node can only receive from or transmit to at most one node at any time-slot $m$. Further, each link is power-controlled. That is, if node-exclusiveness is satisfied, we assume that the possible data rate $R_{e}$ of link $e$ is a result of its power assignment, $p_{e}$. We use $h\left(R_{e}\right)$ to denote the power consumption of supporting data rate of $R_{e}$. It is assumed that $h(\cdot)$ is a nondecreasing, convex function satisfying $h(0)=0$. An example of $h(\cdot)$ is the power-rate relationship in an Additive White Gaussian Noise (AWGN) channel. Other interference models can be incorporated in this formulation, and we will discuss this in Section VI.

Each packet may take multiple hops to be delivered from source to destination. Let $T_{v d}$ denote the long-term average data rate of the flow that needs to be supported from source node $v$ to destination node $d$. We use $D$ to denote the set of destinations.

The joint energy minimization problem is now formulated as follows:

$$
\begin{array}{cl}
\min _{\vec{f}, \vec{R}} \quad \lim _{M \rightarrow \infty} \frac{1}{M} \sum_{m=1}^{M} \sum_{e \in E} h\left(R_{e}(m)\right), \\
\text { subject to } & \vec{R}(m) \text { satisfies node-exclusiveness, } \forall m, \\
& \sum_{d} f_{e}^{d}=\lim _{M \rightarrow \infty} \frac{1}{M} \sum_{m=1}^{M} R_{e}(m), \forall e, \\
& \sum_{e \in N_{o}(v)} f_{e}^{d}-\sum_{e \in N_{i}(v)} f_{e}^{d}-T_{v d} \geq 0 \\
\forall d, \text { and } \forall v \neq d,
\end{array}
$$

where the quantity $f_{e}^{d}$ can be interpreted as the average amount of data rate on link $e$ allocated for destination $d$. The constraints in (2) require that the long-term average data rate, determined by the power allocation, should be able to support the total average data rate $\left(\sum_{d} f_{e}^{d}\right)$ on each link.
The constraints in (3) require that the total outgoing flow of a node should be able to support the total incoming and locally generated flow, for all destinations. Note that we use $\forall d$ as a shorthand notation for all nodes $d$ belonging to $D$, the set of destinations. We will refer to the above problem as Problem $(*)$.

\section{Solution Methodology}

\section{A. Approximating the Energy Minimization Problem}

The optimal solutions developed in [11], [12] attempt to solve Problem $(*)$. However, their solutions contain a scheduling component with high computational complexity. In order to compute at which power and at what time each link $e$ should be activated, these solutions need to solve a complex global optimization problem in each time-slot.

In this paper, in order to obtain a low-complexity and distributed solution, we take a different approach. We first approximate $(*)$ by another optimization problem that is easier to solve. The following Lemma [5] provides the first step in this direction.

Lemma 1: For all time-slot $m$ when link $e$ is activated, the instantaneous data rate $R_{e}(m)$ is independent of $m$ in any power-optimal scheme.

It is worth noting that $R_{e}(m)=R_{e}$ holds when link $e$ is activated. As a result, $\lim _{M \rightarrow \infty} \frac{1}{M} \sum_{m=1}^{M} R_{e}(m)$ is equal to the product of $R_{e}$ and the frequency that link $e$ is activated. Therefore, by (2), the objective function of Problem $(*)$ can now be written as

$$
\sum_{e \in E} \frac{\sum_{d} f_{e}^{d}}{R_{e}} h\left(R_{e}\right),
$$

where $\frac{\sum_{d} f_{e}^{d}}{R_{e}}$ is the fraction of time-slots that link $e$ is activated.

Further, using the results from low-complexity scheduling [13], we have

- Fact 1: In the optimal solution to $(*)$, we must have $\sum_{e \in N(v)} \frac{\sum_{d} f_{e}^{d}}{R_{e}} \leq 1, \forall v \in V$.

- Fact 2: Under the node-exclusive interference model, if $\sum_{e \in N(v)} \frac{\sum_{d} f_{e}^{d}}{R_{e}} \leq \frac{1}{2}-\eta, \forall v \in V$, where $\eta>0$ is an arbitrarily small number, then a maximal schedule can be computed such that each link is activated for $\frac{\sum_{d} f_{e}^{d}}{R_{e}}$ fraction of time-slots [14], [15]. We will discuss more about the role of maximal scheduling in our solution in Section III-D.

Based on these two facts, in the rest of the paper, we will replace the scheduling constraints by

$$
\sum_{e \in N(v)} \frac{\sum_{d} f_{e}^{d}}{R_{e}} \leq \beta, \forall v \in V .
$$


Problem $(*)$ can then be reformulated as the following problem:

$$
\begin{array}{ll}
\min _{\vec{f}, \vec{R}} & \sum_{e \in E} \frac{\sum_{d} f_{e}^{d}}{R_{e}} h\left(R_{e}\right), \\
\text { subject to } & (3) \text { and (4) } \\
& (\vec{f}, \vec{R}) \in X,
\end{array}
$$

where $X=\left\{(\vec{f}, \vec{R}): R_{e} \geq 0, f_{e}^{d} \geq 0, \forall e, d\right\}$. The formulation in (A) is not only easier to solve, but it also produces natural bounds for proving the power efficiency ratio of our solution. Indeed, solving (A) with $\beta=1$ provides a lower bound on the minimum power of $(*)$, while $\beta=\frac{1}{2}-\eta$ provides an upper bound.

Remark: This problem appears in the formulation of [5]. However, our solution is different from this point on. As we mentioned earlier, their solution is a centralized one with an approximation ratio of 3 , while our solution is a distributed one with a better approximation ratio.

\section{B. Handling the Non-Convexity}

In Problem (A), the objective function and constraints (4) are non-convex. Problems of this type are considered to be difficult in general. To overcome this difficulty, the following change of variable is performed:

$$
t_{e}=\frac{\sum_{d} f_{e}^{d}}{R_{e}}, \forall e \in E .
$$

The physical meaning of $t_{e}$ is the fraction of time-slots that link $e$ is activated. It can also be interpreted as the load on link $e$. The latter interpretation seems more appropriate when we deal with $t_{e}(m)$ for each time-slot in the dual solution. Note that $t_{e}=0$ implies that $f_{e}^{d}=0$ for any destination $d$ and any link $e$ : if a link is not activated (or the load is zero), it cannot support any non-zero data rate. The long-term average power consumption $\Theta$ from link $e$ can therefore be defined as

$$
\Theta\left(\vec{f}_{e}, t_{e}\right)= \begin{cases}0, & t_{e}=0 \\ t_{e} h\left(\frac{\sum_{d} f_{e}^{d}}{t_{e}}\right), & t_{e}>0\end{cases}
$$

Using the above notation, Problem (A) can be transformed into

$$
\begin{array}{cl}
\min _{\vec{f}, \vec{t}} & \sum_{e \in E} \Theta\left(\vec{f}_{e}, t_{e}\right), \\
\text { subject to } \quad & \sum_{e \in N(v)} t_{e} \leq \beta, \forall v \in V, \\
& \sum_{e \in N_{o}(v)} f_{e}^{d}-\sum_{e \in N_{i}(v)} f_{e}^{d}-T_{v d} \geq 0, \\
\forall d, \text { and } \forall v \neq d, & (\vec{f}, \vec{t}) \in Y,
\end{array}
$$

where $Y=\left\{(\vec{f}, \vec{t}): f_{e}^{d} \geq 0, \forall e, d ; 0 \leq t_{e} \leq 1, \sum_{d} f_{e}^{d} \leq\right.$ $a t_{e}, \forall e$, for some $\left.a \geq 0\right\}$.

Remark: The condition $\sum_{d} f_{e}^{d} \leq a t_{e}$, for some $a \geq 0$, in the definition of $Y$ is required to guarantee that the optimal solution from Problem (B) can be transformed to the optimal solution of Problem (A). Without this condition, Problem (B) can be trivially solved by setting $\vec{t}=0$ and $\vec{f}$ large enough for the flow constraint. Such a solution cannot be transformed to an optimal solution of Problem (A), since the instantaneous data rate $\vec{R}$ must be finite.

To show that there is no duality gap in solving Problem (B), we need the following Lemma:

\section{Lemma 2: Let}

$$
\theta(f, t)= \begin{cases}0, & t=0 \\ t h\left(\frac{f}{t}\right), & t>0\end{cases}
$$

If $h(\cdot)$ is a convex function on $\mathbb{R}^{+}$, then $\theta(f, t)$ is also convex on $C=\bigcup_{a \in[0,+\infty)}\{(f, t): 0 \leq f \leq a t, t \geq 0\}$.

In our online technical report [16], we have included a proof of Lemma 2.

Remark: A function of the form $g(f, t)=t h\left(\frac{f}{t}\right)$, where $t>0$ is known as the perspective of function $h(f)$. As shown in [17, p89], the perspective operation is one of the transformations that preserve convexity. The transformation in the above Lemma includes the $t=0$ case, and therefore can be viewed as a slightly generalized version of the perspective operation. Our proof in [16] takes a different approach than [17].

Note that $\theta$ is non-convex over the entire $\mathbb{R}^{+} \times \mathbb{R}^{+}$. However, the convexity of $\theta$ over $C$ is enough for our purpose, since $(\vec{f}, \vec{t}) \in Y$ in Problem (B). Due to Lemma 2, the objective function $\Theta(\vec{f}, \vec{t})$ of (B) is convex, and the entire problem is a convex program. We can then use the duality approach to solve the problem.

\section{Distributed Algorithm Based on Lagrange Duality}

To use the duality approach to solve the above problem, we first form the Lagrangian:

$$
\begin{aligned}
& L(\vec{f}, \vec{t}, \vec{\mu}, \vec{q}) \\
= & \sum_{e \in E} \Theta\left(\vec{f}_{e}, t_{e}\right)+\sum_{v \in V} \mu_{v}\left(\sum_{e \in N(v)} t_{e}-\beta\right) \\
& -\sum_{v, d} q_{v}^{d}\left(\sum_{e \in N_{o}(v)} f_{e}^{d}-\sum_{e \in N_{i}(v)} f_{e}^{d}-T_{v d}\right),
\end{aligned}
$$

where $\vec{\mu} \geq 0$ and $\vec{q} \geq 0$ are the Lagrange multipliers, and $\overrightarrow{f_{e}}=\left\{f_{e}^{d}\right\}_{d \in D}$. For ease of notation, we define $q_{d}^{d}=0$, for all $d$. By rearranging the order of summation, the above equation can be transformed into the following:

$$
\begin{aligned}
L(\vec{f}, \vec{t}, \vec{\mu}, \vec{q}) & \sum_{e \in E} c_{e}\left(\vec{f}_{e}, t_{e}\right)-\beta \sum_{v} \mu_{v}+\sum_{v, d}\left(q_{v}^{d} T_{v d}\right),
\end{aligned}
$$


where

$$
\begin{aligned}
c_{e}\left(\vec{f}_{e}, t_{e}\right)= & \Theta\left(\vec{f}_{e}, t_{e}\right)+\left(\mu_{x(e)}+\mu_{r(e)}\right) t_{e} \\
& -\sum_{d}\left(q_{x(e)}^{d}-q_{r(e)}^{d}\right) f_{e}^{d},
\end{aligned}
$$

and $x(e)$ and $r(e)$ are the transmission node and reception node of link $e$, respectively.

The dual objective function is

$$
\begin{aligned}
& D(\vec{\mu}, \vec{q}) \\
= & \min _{(\vec{f}, \vec{t}) \in Y} L(\vec{f}, \vec{t}, \vec{\mu}, \vec{q}), \\
= & \sum_{e \in E}\left[\min _{\left(\vec{f}_{e}, t_{e}\right) \in Y_{e}} c_{e}\left(\vec{f}_{e}, t_{e}\right)\right]-\beta \sum_{v} \mu_{v}+\sum_{v, d}\left(q_{v}^{d} T_{v d}\right),
\end{aligned}
$$

where $Y_{e}$ denotes the constraint set on link $e$, namely

$$
\begin{aligned}
Y_{e}= & \left\{\left(\vec{f}_{e}, t_{e}\right): 0 \leq f_{e}^{d} \leq H, \forall d ; 0 \leq t_{e} \leq 1 ;\right. \\
& \left.\sum_{d} f_{e}^{d} \leq a t_{e}, \text { for some } a \geq 0\right\} .
\end{aligned}
$$

To ensure convergence, we have added $f_{e}^{d} \leq H$, where $H$ is a large constant to be determined later. See the proof of Theorem 4 (in Appendix) for details. The minimization of the Lagrangian can now be decomposed into a minimization on each link. Note that all the information needed in minimizing $c_{e}\left(\vec{f}_{e}, t_{e}\right)$ is local to link $e$.

The dual optimization problem is

$$
\max _{\vec{\mu} \geq 0, \vec{q} \geq 0} D(\vec{\mu}, \vec{q}) .
$$

As mentioned before, the convexity of $\Theta(\vec{f}, \vec{t})$ can be inferred from Lemma 2. Based on this, the following Theorem [16] establishes the relationship between the primal problem (B) and the dual problem (C) above. Due to space constraint, we state it without proof.

\section{Theorem 3: (Strong Duality)}

Problem (B) is feasible and its optimal value $\Theta^{*}$ is finite. Further, there is no duality gap: $\Theta^{*}$ can be found by solving its dual optimization problem.

The next step is to solve the dual problem in a distributed fashion. We can show that $D(\vec{\mu}, \vec{q})$ is convex and its subgradient is given by

$$
\begin{aligned}
\frac{\partial D}{\partial \mu_{v}} & =\sum_{e \in N(v)} t_{e}-\beta, \\
\frac{\partial D}{\partial q_{v}^{d}} & =-\left[\sum_{e \in N_{o}(v)} f_{e}^{d}-\sum_{e \in N_{i}(v)} f_{e}^{d}-T_{v d}\right] .
\end{aligned}
$$

We can then use the following subgradient method to solve the dual problem.

\section{Distributed Energy Minimization Algorithm}

At each iteration $m$,
1) At link $e$, the data rate $\vec{f}_{e}$ and the link assignment $t_{e}$ are determined by:

$$
\begin{aligned}
\left(\vec{f}_{e}(m), t_{e}(m)\right) \\
=\underset{\left(\vec{f}_{e}, t_{e}\right) \in Y_{e}}{\operatorname{argmin}} c_{e}\left(\vec{f}_{e}, t_{e}, m\right) \\
=\underset{\left(\vec{f}_{e}, t_{e}\right) \in Y_{e}}{\operatorname{argmin}}\left[\Theta\left(\vec{f}_{e}, t_{e}\right)+\left(\mu_{x(e)}(m)+\mu_{r(e)}(m)\right) t_{e}\right. \\
\left.\quad-\sum_{d}\left(q_{x(e)}^{d}(m)-q_{r(e)}^{d}(m)\right) f_{e}^{d}\right] \cdot \text { (15) }
\end{aligned}
$$

2) At node $v$, the dual variables are updated by:

$$
\begin{aligned}
\mu_{v}(m+1)= & \left\{\mu_{v}(m)+\alpha_{m}\left[\sum_{e \in N(v)} t_{e}(m)-\beta\right]\right\}_{(16}^{+} \\
q_{v}^{d}(m+1)= & \left\{q_{v}^{d}(m)-\alpha_{m}\left[\sum_{e \in N_{o}(v)} f_{e}^{d}(m)\right.\right. \\
& \left.\left.-\sum_{e \in N_{i}(v)} f_{e}^{d}(m)-T_{v d}\right]\right\}^{+}
\end{aligned}
$$

Remark: At each iteration $m$, the data rate and schedule are chosen to minimize the total $\operatorname{cost} c_{e}$, given the current implicit costs $(\vec{\mu}, \vec{q})$. To maximize the dual objective function, the dual variables are updated according to the subgradient ascent procedure in (16) and (17), where $\left\{\alpha_{m}\right\}$ are the stepsizes. In the above algorithm, we use the same stepsize for $\vec{\mu}$ and $\vec{f}$ at each iteration. This is simply for ease of notation: the stepsizes can be different for each dual variable. Since the primal cost function is not strictly convex, the dual function may not be differentiable. The stepsizes need to be chosen carefully to guarantee the convergence of the dual variables. We will discuss this issue in the Section IV.

The above exercise of using Lagrange duality is standard. Nonetheless, there are some questions that are not answered by the above algorithm alone. One of them is how the algorithm maps to different protocol components. We will now study this problem.

\section{Mapping to Network Protocol Components}

To decide the routing and scheduling, each link solves (15) by minimizing $c_{e}\left(\vec{f}_{e}, t_{e}, m\right)$. Note that there are three components in $c_{e}\left(\overrightarrow{f_{e}}, t_{e}, m\right)$ :

- The term $\Theta\left(\vec{f}_{e}, t_{e}\right)$ is the power cost, i.e., the power consumption of supporting an average data rate of $\vec{f}_{e}$ while link $e$ being activated for $t_{e}$ fraction of time.

- The term $\left(\mu_{x(e)}(m)+\mu_{r(e)}(m)\right) t_{e}$ is the scheduling cost. We will show that $t_{e}(m)$ will either be 0 or 1 in order for link $e$ to minimize $c_{e}\left(\vec{f}_{e}, t_{e}\right)$. If $t_{e}(m)$ is chosen to be 1 , then $\mu_{x(e)}(m+1) \geq \mu_{x(e)}(m)$ from (16): scheduling the transmission on link $e$ gives rise to increased (or at least the same) scheduling costs on the 
transmission/reception nodes. If $t_{e}(m)$ is chosen to be 0 , then $\mu_{x(e)}(m+1) \leq \mu_{x(e)}(m)$ : an idle link gives the system more flexibility in scheduling other links, which leads to a potential decrease in the scheduling costs on the transmission/reception nodes.

- The term $\sum_{d}\left(q_{x(e)}^{d}(m)-q_{r(e)}^{d}(m)\right) f_{e}^{d}$ is the utility of supporting the total data rate on link $e$. The term $q_{v}^{d}$ can be interpreted as an approximation of the scaled version of the queue length at node $v$ for destination $d$ if constant stepsizes are used [14], [18]. As in (17), if $f_{e}^{d}(m)>0$, it helps to reduce the workload at the transmission node, but add to the workload at the reception node.

The following transformation leads to a better understanding of the minimization of $c_{e}\left(\vec{f}_{e}, t_{e}\right)$ :

$$
f_{e}^{d}(m)=R_{e}^{d}(m) t_{e}(m),
$$

where $R_{e}^{d}(m)$ is the instantaneous data rate allocated on link $e$ for destination $d$, and $f_{e}^{d}(m)$ is the resultant average data rate when transmitting for $t_{e}(m)$ fraction of the time. Substitute the above equation into $c_{e}\left(\vec{f}_{e}, t_{e}, m\right)$, we have (all time index dropped for ease of notation):

$$
c_{e}\left(\vec{f}_{e}, t_{e}\right)=t_{e} l_{e}\left(\vec{R}_{e}\right),
$$

where $l_{e}\left(\vec{R}_{e}\right)$ is defined as

$$
\begin{aligned}
l_{e}\left(\vec{R}_{e}\right)= & h\left(\sum_{d} R_{e}^{d}\right)+\left(\mu_{x(e)}+\mu_{r(e)}\right) \\
& -\sum_{d}\left[\left(q_{x(e)}^{d}-q_{r(e)}^{d}\right) R_{e}^{d}\right] .
\end{aligned}
$$

Since $t_{e} \geq 0$, to minimize (19), we should first minimize $l_{e}\left(\vec{R}_{e}\right)$ as a function of $\vec{R}_{e}$. Note that function $h(\cdot)$ takes as input parameter the sum of the data rates allocated for all the destinations on this link. In other words, from the viewpoint of power consumption, it is indifferent which destination the data rate is allocated for, as long as the total data rate is the same. As a result, the minimum of $l_{e}\left(\vec{R}_{e}\right)$ is attained when all the data rates are allocated to the destination with the maximum positive backlog difference. Let

$$
\hat{d}=\underset{d}{\operatorname{argmax}}\left(q_{x(e)}^{d}-q_{r(e)}^{d}\right),
$$

then $R^{\hat{d}}>0$ if $\left(q_{x(e)}^{\hat{d}}-q_{r(e)}^{\hat{d}}\right)>0$, and $R_{e}^{d}=0$ for all $d \neq \hat{d}$. With this observation, the minimization of $l_{e}\left(\vec{R}_{e}\right)$ is relatively simple. For example, if $h(x)=e^{x}-1$, and $\max _{d}\left(q_{x(e)}^{d}-\right.$ $\left.q_{r(e)}^{d}\right)>0$, then $R_{e}^{\hat{d}}=\left[\log \left(q_{x(e)}^{\hat{d}}-q_{r(e)}^{\hat{d}}\right)\right]^{+}$, and $R_{e}^{d}=0$ for all other destination $d$.

Now that $\vec{R}_{e}$ has been chosen to minimize $l_{e}\left(\vec{R}_{e}\right)$ in (20), the next step is to determine the value of $t_{e}$ over the interval $[0,1]$ to minimize $c_{e}\left(\vec{f}_{e}, t_{e}\right)=t_{e} l_{e}\left(\vec{R}_{e}\right)$. Clearly, the optimal $t_{e}$ value is

$$
\hat{t}_{e}= \begin{cases}1, & \text { if } \min _{\vec{R}_{e}} l_{e}\left(\vec{R}_{e}\right) \leq 0, \\ 0, & \text { if } \min _{\vec{R}_{e}} l_{e}\left(\vec{R}_{e}\right)>0 .\end{cases}
$$

Remark: There are two possible scenarios where $\hat{t}_{e}=0$ (no load is assigned in this time-slot):
- All backlog differences $\left(q_{x(e)}^{d}-q_{r(e)}^{d}\right)$ are negative, which means the utility does not increase by transporting data to next hop on this link for any destination.

- Although some backlog differences $\left(q_{x(e)}^{d}-q_{r(e)}^{d}\right)$ are positive, as in (20), the utility of transporting data to next hop is not large enough to outweigh the importance of saving more energy (the $h\left(\sum_{d} R_{e}^{d}\right)$ term) and/or the need to gain more flexibility in scheduling (the $\left(\mu_{x(e)}+\mu_{r(e)}\right)$ term). As a result, optimal $l_{e}\left(\vec{R}_{e}\right)$ is non-negative, and no load is assigned to this link.

To summarize, the minimization of $c_{e}\left(\vec{f}_{e}, t_{e}\right)$ on each link naturally translates into the following protocol components:

1) Routing: Choose only the flow $\hat{d}$ with maximum positive backlog difference (cf. (21)). This is the flow that should receive service.

2) Power control: Choose $\vec{R}_{e}$ to minimize $l_{e}\left(\vec{R}_{e}\right)$. This is the power link $e$ should use.

3) Link assignment: Choose $t_{e}$ to minimize $t_{e} l_{e}\left(\vec{R}_{e}\right)$ in such a way that $t_{e}$ takes its maximum value 1 if the optimal $l_{e}\left(\vec{R}_{e}\right)$ is less than or equal to 0 ; and 0 otherwise. This determines the amount of time link $e$ should be on.

4) Maximal Scheduling: As mentioned before, given the fraction of up-time, or load, on each link, a scheduling component is needed to determine the exact time-slots link $e$ should be on. In other words, the link assignment from previous step may have scheduling conflicts, and therefore cannot be used as a transmission schedule as it is. A scheduling component can resolve such conflicts by delaying the transmission by a certain number of time-slots. Clearly, given the link assignment $\vec{t}(m)$, the delay can be different for different links. With the nodeexclusive interference model, by choosing $\beta=\frac{1}{2}-\eta$, it can be shown that a scheduling policy based on maximal matching can stabilize the system (i.e., the delay of transmission mentioned above is bounded) [14], [15], [19]-[22]. Maximal schedules such as this can be implemented in a distributed fashion. We refer readers to [13] for more details on the distributed implementation of maximal schedules.

As we have seen thus far, the duality approach exploits the problem structure and decomposes the primal problem into sub-problems on each link that are much simpler. In addition, some of the quantities produced/monitored by this algorithm actually help network engineering. For instance, the Lagrange multiplier $q_{v}^{d}$ can also be interpreted as the shadow price of the corresponding constraint. If a small change occurs in the amount of supported traffic from node $v$ to node $d, q_{v}^{d}$ measures the sensitivity of the optimal power consumption with respect to this perturbation. In a different networking setting where the network has some control over the traffic matrix $\vec{T}$, information such as $\vec{q}$ can be used as guidelines to optimize power consumption. 


\section{Performance Analysis}

In this section, first we are interested in the following question: Under what condition do the dual variables in the Distributed Energy Minimization algorithm converge?

Furthermore, as we have seen in Section III, there is no guarantee that $(\vec{f}(m), \vec{t}(m))$ will converge, even if the dual variables converge. For example, $t_{e}(m)$ is either 0 or 1 from the distributed dual algorithm. A natural question to ask then is the following: In what sense is the primal solution optimal? The two Theorems below answer both of the above questions from two different perspectives.

\section{A. Convergence Result with Constant Stepsizes}

The following theorem establishes the stability and the optimality of the proposed dual algorithm.

\section{Theorem 4: (Stability and Optimality)}

Let the stepsizes in the Distributed Energy Minimization algorithm be constant, i.e., $\alpha_{m}=h$, for all $m$. Let $\Phi$ be the set of $(\vec{\mu}, \vec{q})$ that maximizes $D(\vec{\mu}, \vec{q})$, and $d((\vec{\mu}, \vec{q}), \Phi)=$ $\min _{\left(\vec{\mu}^{*}, \vec{q}^{*}\right) \in \Phi}\left\|(\vec{\mu}, \vec{q})-\left(\vec{\mu}^{*}, \vec{q}^{*}\right)\right\|$. Given any $\varepsilon>0$, there exists some $h_{0}>0$ such that, for any $h \leq h_{0}$ and any initial implicit costs $\left(\vec{\mu}_{0}, \vec{q}_{0}\right)$, there exists a time $M_{0}$ such that for all $m>$ $M_{0}$,

$$
d((\vec{\mu}(m), \vec{q}(m)), \Phi)<\varepsilon,
$$

and

$$
\limsup _{m \rightarrow \infty} \frac{1}{m} \sum_{\tau=1}^{m} \sum_{e \in E} \Theta\left(\vec{f}_{e}(\tau), t_{e}(\tau)\right)<\Theta^{*}+\varepsilon .
$$

The proof of Theorem 4 is included in the Appendix.

The above Theorem shows that, when stepsizes are small, the dual variables eventually converge to within a small neighborhood of the optimal dual solution. Further, the power consumption from the distributed algorithm is asymptotically optimal: although the primal variables $(\vec{f}(m), \vec{t}(m))$ may not converge, by using $(\vec{f}(m), \vec{t}(m))$ for each time-slot $m$, the long-term average of the resultant power consumption is arbitrarily close to the optimal power consumption.

\section{B. Optimality of Primal Variables}

As shown in Theorem 4, with constant stepsizes, the dual variables eventually converge to within a small neighborhood of the optimal dual solution. When appropriate diminishing stepsizes are used in the dual algorithm, the following Theorem shows that the entire sequence of the dual variables converges to one point. In this case, we also obtain a different interpretation of the primal optimality.

\section{Theorem 5: (Primal Optimality with Diminishing Step-} sizes)

(a) Let the stepsizes $\left\{\alpha_{m}\right\}$ in the Distributed Energy Minimization algorithm satisfy the following conditions:

$$
\sum_{m=1}^{\infty} \alpha_{m}^{2}<+\infty, \quad \sum_{m=1}^{\infty} \alpha_{m}=+\infty
$$

Then for any nonnegative starting point $\left(\vec{\mu}_{0}, \vec{q}_{0}\right)$, the dynamics of the Distributed Energy Minimization algorithm converges to the optimal value of Problem (B):

$$
\begin{aligned}
& \lim _{m \rightarrow \infty}(\vec{\mu}(m), \vec{q}(m))=\left(\vec{\mu}^{*}, \vec{q}^{*}\right), \\
& \lim _{m \rightarrow \infty} D(\vec{\mu}(m), \vec{q}(m))=\Theta^{*},
\end{aligned}
$$

where $\left(\vec{\mu}^{*}, \vec{q}^{*}\right)$ is a maximum point of $D(\vec{\mu}, \vec{q})$.

(b) Let the stepsizes $\left\{\alpha_{m}\right\}$ be chosen as

$$
\alpha_{m}=\frac{\kappa}{m+\rho},
$$

where $\kappa$ and $\rho$ are some positive scalars (note that the above definition of $\left\{\alpha_{m}\right\}$ satisfies the conditions (25)). If the longterm average of the vector $(\vec{f}(m), \vec{t}(m))$ converges, i.e.,

$$
\lim _{M \rightarrow \infty} \frac{1}{M} \sum_{m=1}^{M} \vec{f}(m)=\vec{f}^{*}, \quad \lim _{M \rightarrow \infty} \frac{1}{M} \sum_{m=1}^{M} \vec{t}(m)=\vec{t}^{*},
$$

then the time-average version of $(\vec{f}(m), \vec{t}(m))$ is the optimal solution to Problem (B): $\sum_{e \in E} \Theta\left(\overrightarrow{f_{e}^{*}}, t_{e}^{*}\right)=\Theta^{*}$.

The proof of Theorem 5 is included in our online technical report [16].

Remark: In the Distributed Energy Minimization algorithm, $t_{e}(m)$, for example, can only be 0 or 1 . From this perspective, our solution carries the same flavor as some of the related work [11], [14], namely, in the optimal solution, the resource, be it power or fraction of up-time, is used to the maximum extent, if the link is activated for the current time-slot. However, one would expect the optimal solution of $t_{e}$ to Problem (B) to be a number anywhere from 0 to $\beta$ for most of the links in a typical setting (cf. (8)). Theorem 5 reconciles the difference between these two viewpoints. Although $(\vec{f}(m), \vec{t}(m))$ is not a continuous mapping from the underlying converging implicit costs (dual variables $(\vec{\mu}, \vec{q})$ ), as long as the long-term average of primal variables $(\vec{f}(m), \vec{t}(m))$ converges, the limit is an optimal solution to the primal problem.

\section{Power-Efficiency Ratio}

Setting $\beta=1$ or $\beta=\frac{1}{2}-\eta$ in (4) gives necessary or sufficient conditions for schedulability, in terms of stabilizing the overall queueing system [14], [23]. From the necessary condition of $\beta=1$, it is evident that the maximum loss in throughput under the node exclusive interference model is $\frac{1}{2}-$ $\eta$. This is the throughput loss ratio in approximating (*) using Problem (B) with $\beta=\frac{1}{2}-\eta$.

To derive the approximation ratio of our algorithm, the throughput loss needs to be translated into power loss. We apply the same first order approximation of the rate-power function as in [5]. More specifically, in an AWGN channel, the total power consumption can be approximated as $\sum_{e} \frac{B_{e}}{t_{e}}$, where $B_{e}$ is a flow-related constant independent of $t_{e}$. Let $(\vec{f}, \vec{t})$ be the optimal solution to Problem (B) with $\beta=1$. It is evident that $\left(\vec{f}, \frac{\vec{t}}{2+\varepsilon}\right)$, where $\varepsilon$ is a small positive constant, is a feasible solution to Problem (B) with $\beta=\frac{1}{2}-\eta$. This feasible solution results in a power consumption that is at most $(2+\varepsilon)$ 
TABLE I

THE TWO FLOWS SUPPORTED BY THE NETWORK

\begin{tabular}{|c|c|c|c|c|}
\hline & source & destination & data rate & paths \\
\hline flow 1 & 1 & 7 & $250 \mathrm{kbps}$ & $1-7,1-2-7$ \\
\hline flow 2 & 3 & 6 & $500 \mathrm{kbps}$ & $3-2-6,3-4-5-6$ \\
\hline
\end{tabular}

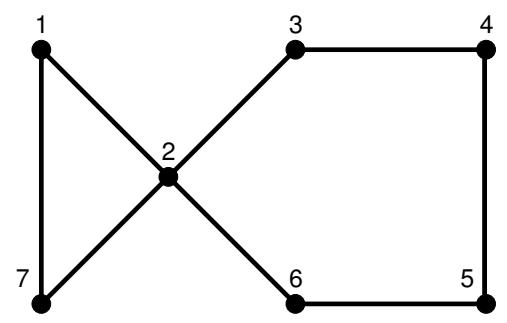

Fig. 1. Network topology

that of the optimal value with $\beta=1$. Since the optimal value of Problem (B) with $\beta=1$ is a lower bound on the minimum power from $(*)$, we conclude that the power-efficiency ratio of our algorithm is upper-bounded by $(2+\varepsilon)$.

\section{Numerical Results}

In this illustrative example, we consider a 7-node network, whose topology is depicted in Figure 1. The power-rate function is of the following form:

$$
R_{e}=W \log _{2}\left[1+\frac{\sigma_{e} p_{e}}{N_{0} W}\right],
$$

where $W=1.0 \mathrm{MHz}$ is the available bandwidth, $\sigma_{e}=1.6 \times$ $10^{-13}$ is the channel gain of link $e, N_{0}=1.6 \times 10^{-18} \mathrm{~mW} / \mathrm{Hz}$ is the noise spectral density, $p_{e}$ is the transmission power, and $R_{e}$ is the resultant instantaneous data rate of link $e$. This network supports two flows, as shown in Table I.

The node-exclusive interference model is considered, and $\beta=\left(0.5-10^{-4}\right)$ in Problem (A). The length of each timeslot is 1 second. The results reported in this section are the average over a moving time window of length 120 seconds.

To show that the joint energy minimization algorithm can adapt to variations in the input parameters, we apply the following changes in the system setting. At time $t=4000 \mathrm{~s}$, the channel gain $\sigma_{(1,7)}$ of the direct link between node 1 and node 7 is decreased from $1.6 \times 10^{-13}$ to $0.4 \times 10^{-13}$. At time $t=8000$ s, the data rate of flow 2 (from node 3 to node 6 ) is reduced from $500 \mathrm{kbps}$ to $250 \mathrm{kbps}$.

For each setting, offline computation is carried out to find the optimal value of Problem (A), which is given by the dashed line in Figure 2. The power consumption from the proposed algorithm is shown as the solid line in the same figure. This simulation result shows that the Distributed Energy Minimization algorithm is capable of computing the optimal solution to Problem (A) in a distributed manner, and

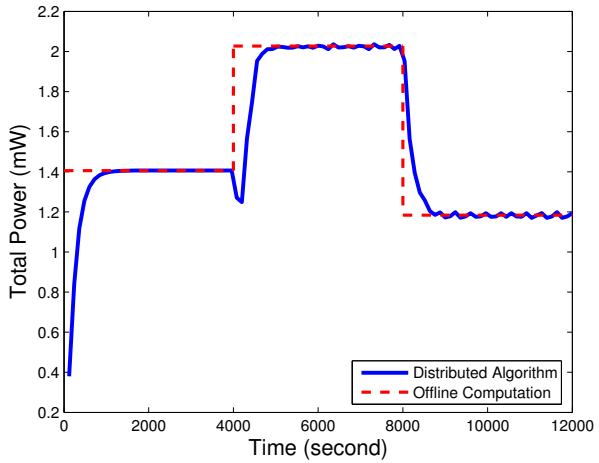

Fig. 2. Power consumption from distributed algorithm and offline computation

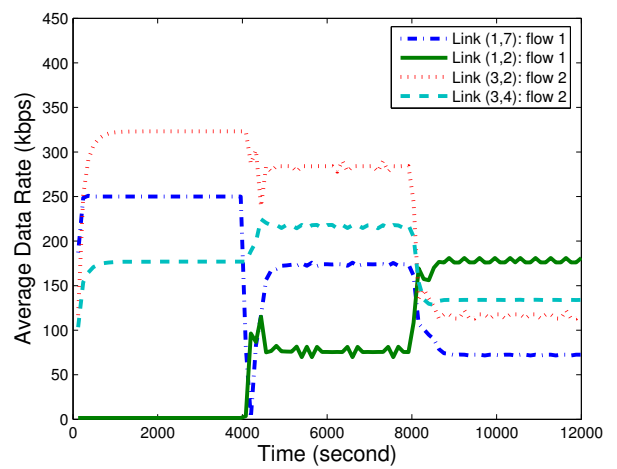

Fig. 3. Average data rates for different flows on four links

automatically tracking the optimal operating point once the system parameters change.

Figure 3 shows the average data rates $f_{e}^{d}$ for different flows on four links. We now take a closer look at the routing of the flows.

- In the initial state, flow 1 concentrates on the minimum energy path, namely, link $(1,7)$.

- At $t=4000$ s, the channel gain $\sigma_{(1,7)}$ reduces by $75 \%$, and part of flow 1 is shifted to path $1-2-7$. Since the scheduling capacity of node 2 is saturated, a larger percentage of flow 2 is then routed through path $3-4-$ $5-6$.

- At $t=8000 \mathrm{~s}$, the traffic that the network has to support between node 3 and node 6 reduces (flow 2 is reduced to $250 \mathrm{kbps}$ ). As a consequence, part of the scheduling capacity of node 2 is freed, and more of flow 1 takes path $1-2-7$ to reduce the overall power consumption.

The above example shows that the interaction between routing, scheduling, and power control is relatively complex even in a wireless network of small size. The correct way to deal with such interaction is difficult to summarize into general approaches such as minimum energy approach or load balancing approach. In our Distributed Energy Minimization algorithm, as shown in Figure 2 and Figure 3, low-complexity 
and distributed operation on each link accomplishes this joint optimization even in networks with non-stationarity.

\section{CONCLUSION}

In this paper, we propose a joint power control, link scheduling, and routing algorithm to minimize the power consumption in multi-hop wireless networks. The known crosslayer solution to this problem is centralized, and with high computational complexity. In contrast, our algorithm is distributed, and with low computational complexity. We establish the power efficiency ratio of our distributed solution, and show that it is provably tighter than the power efficiency ratio of a centralized solution in the literature. We map our solution to corresponding components of the cross-layer control protocols, and discuss the implication of our result on network protocol design. The stability and optimality of our solution is verified via simulations.

In this work, we focus on the well-studied node exclusive interference model. In a more general interference model, we can define $\Gamma_{e}$ to be the set of links that interfere with link $e$. (The node-exclusive model can be viewed as a special case with $\Gamma_{e}=N(x(e)) \bigcup N(r(e))$.) Let $\omega_{e}$ denote the maximum number of links that can be scheduled simultaneously in $\Gamma_{e}$, and $\omega_{\max }=\max _{e} \omega_{e}$. It can be shown that a distributed maximal schedule can stabilize the system while the throughput is reduced by at most a factor of $1 / \omega_{\max }$. In this case, the left hand side of the scheduling constraint (8) in our problem formulation need to be modified accordingly [19][22]. Nonetheless, the general methodology presented in this paper can be carried through to this type of interference model.

\section{APPENDIX}

\section{Proof of Theorem 4:}

The proof technique here is similar to [12].

We first show the boundedness of the subgradient of the dual objective function. In (13), we choose $H \geq|V|^{2} \max _{v \neq d} T_{v d}$, where $|V|$ is the number of nodes in the network. Let $\bar{Y}$ denote the Cartesian product of all $Y_{e}$ in (13). It is clear that $\bar{Y} \subset Y$, where $Y$ is defined in Problem (B). Note that the introduction of $H$ does not change the solution. The optimal solution $\left(\overrightarrow{f^{*}}, \overrightarrow{t^{*}}\right)$ to Problem (B) is in $\bar{Y}$ for the following reason: $\Theta$ is a non-decreasing function of $f_{e}^{d}$, and therefore any $(\vec{f}, \vec{t})$ with $f_{e}^{d}>H$ for some $d$ and $e$ is clearly suboptimal even if all the traffic is routed through link $e$. So the solution generated from the Distributed Energy Minimization algorithm coincides with the optimal solution to Problem (B). The benefit from enforcing such an upper bound on $f_{e}^{d}$ is that the subgradient of the dual objective function is then bounded.

Given the subgradient of the dual objective function is bounded, it can be shown [24] that, given any $\varepsilon>0$, there exists some $h_{1}>0$ such that, for any $h \leq h_{1}$ and any initial implicit costs $\left(\vec{\mu}_{0}, \vec{q}_{0}\right)$, there exists a time $M_{0}$ such that (23) holds for all $m>M_{0}$. Therefore, as time index $m$ increases, $(\vec{\mu}(m), \vec{q}(m))$ converges to within a small neighborhood of the set of the maximizer of $D(\vec{\mu}, \vec{q})$ if the constant stepsize is chosen to be small enough. This implies that the sequence $\{(\vec{\mu}(m), \vec{q}(m))\}_{m}$ is bounded.

To show (24), we consider the following Lyapunov function:

$$
V(\vec{\mu}(m), \vec{q}(m))=\frac{1}{2} \sum_{v, d}\left[q_{v}^{d}(m)\right]^{2}+\frac{1}{2} \sum_{v} \mu_{v}^{2}(m) .
$$

The subgradient of $D(\vec{\mu}, \vec{q})$ at time-slot $m$ can be written as

$$
\begin{aligned}
\Delta \mu_{v}(m) & =\sum_{e \in N(v)} t_{e}(m)-\beta, \\
\Delta q_{v}^{d}(m) & =-\left[\sum_{e \in N_{o}(v)} f_{e}^{d}(m)-\sum_{e \in N_{i}(v)} f_{e}^{d}(m)-T_{v d}\right] .
\end{aligned}
$$

Using the above notation, the one-step drift of the Lyapunov function can be calculated as follows:

$$
\begin{aligned}
& V(\vec{\mu}(m+1), \vec{q}(m+1))-V(\vec{\mu}(m), \vec{q}(m)) \\
= & \frac{1}{2} \sum_{v}\left\{\left[\left(\mu_{v}(m)+h \Delta \mu_{v}(m)\right)^{+}\right]^{2}-\mu_{v}^{2}(m)\right\} \\
& +\frac{1}{2} \sum_{v, d}\left\{\left[\left(q_{v}^{d}(m)+h \Delta q_{v}^{d}(m)\right)^{+}\right]^{2}-\left[q_{v}^{d}(m)\right]^{2}\right\} \\
& (\text { from }(16) \text { and (17)) } \\
\leq & \frac{1}{2} \sum_{v}\left\{\left[\mu_{v}(m)+h \Delta \mu_{v}(m)\right]^{2}-\mu_{v}^{2}(m)\right\} \\
& +\frac{1}{2} \sum_{v, d}\left\{\left[q_{v}^{d}(m)+h \Delta q_{v}^{d}(m)\right]^{2}-\left[q_{v}^{d}(m)\right]^{2}\right\} \\
\leq & h \sum_{v} \mu_{v}(m) \Delta \mu_{v}(m)+h \sum_{v, d} q_{v}^{d}(m) \Delta q_{v}^{d}(m)+h^{2} W,
\end{aligned}
$$

where $W$ is a constant large enough to guarantee the last inequality. It is possible to choose such a constant since the subgradient of $D(\vec{\mu}, \vec{q})$ is bounded. Adding $h \sum_{e \in E} \Theta\left(\overrightarrow{f_{e}}(m), t_{e}(m)\right)$ to both sides of the above formula, we have

$$
\begin{aligned}
& V(\vec{\mu}(m+1), \vec{q}(m+1))-V(\vec{\mu}(m), \vec{q}(m)) \\
& +h \sum_{e \in E} \Theta\left(\vec{f}_{e}(m), t_{e}(m)\right) \\
\leq \quad & h\left[\sum_{e \in E} \Theta\left(\vec{f}_{e}(m), t_{e}(m)\right)+\sum_{v} \mu_{v}(m) \Delta \mu_{v}(m)\right. \\
& \left.+\sum_{v, d} q_{v}^{d}(m) \Delta q_{v}^{d}(m)\right]+h^{2} W \\
= & h D(\vec{\mu}(m), \vec{q}(m))+h^{2} W \\
& (\text { from }(12) \text { and }(15)) \\
\leq & h D^{*}+h^{2} W \\
= & h \Theta^{*}+h^{2} W,
\end{aligned}
$$

where $D^{*}$ is the maximum dual value, which is also equal to the minimum power $\Theta^{*}$ from the primal problem. 
Summing the above inequality over $m=1,2, \ldots, M$, and dividing both sides by $M$, we have

$$
\begin{aligned}
& \frac{V(\vec{\mu}(M+1), \vec{q}(M+1))-V(\vec{\mu}(1), \vec{q}(1))}{M} \\
& +\frac{h}{M} \sum_{m=1}^{M} \sum_{e \in E} \Theta\left(\vec{f}_{e}(m), t_{e}(m)\right) \\
\leq & h \Theta^{*}+h^{2} W .
\end{aligned}
$$

Note that all $V(\cdot)$ is bounded since the sequence $\{(\vec{\mu}(m), \vec{q}(m))\}_{m}$ is bounded. For any given $\varepsilon>0$, the first term on the LHS of (30) can be bounded by $\varepsilon / 2$ for $M$ large enough:

$$
\frac{1}{M} \sum_{m=1}^{M} \sum_{e \in E} \Theta\left(\vec{f}_{e}(m), t_{e}(m)\right) \leq \Theta^{*}+h W+\frac{\varepsilon}{2} .
$$

For any given $\varepsilon>0$, there exist some $h_{2}>0$ such that $h W<\varepsilon / 2$ for all $h<h_{2}$. Under this condition, the following is evident:

$$
\limsup _{M \rightarrow \infty} \frac{1}{M} \sum_{m=1}^{M} \sum_{e \in E} \Theta\left(\vec{f}_{e}(m), t_{e}(m)\right)<\Theta^{*}+\varepsilon .
$$

Let $h_{0}=\min \left(h_{1}, h_{2}\right)$, and Theorem 4 follows.

\section{REFERENCES}

[1] Intel Research Laboratory at Berkeley, "Habitat monitoring on great duck island," 2002, available on http://www.greatduckisland.net/.

[2] T. P. Ruggaber and J. W. Talley, "Detection and control of combined sewer overflow events using embedded sensor network technology," in World Water Congress, Anchorage, Alaska, May 2005.

[3] J. Luo and J.-P. Hubaux, "A survey of inter-vehicle communication," EPFL, Lausanne, Switzerland, Tech. Rep., 2004.

[4] I. F. Akyildiz, X. Wang, and W. Wang, "Wireless mesh networks: a survey," Computer Networks, vol. 47, no. 4, pp. 445-487, 2005.

[5] R. Bhatia and M. Kodialam, "On power efficient communication over multi-hop wireless networks: Joint routing, scheduling and power control,' in IEEE INFOCOM'04, Hong Kong, Mar. 2004.

[6] K. Kar, M. Kodialam, T. V. Lakshman, and L. Tassiulas, "Routing for network capacity maximization in energy-constrained ad-hoc networks," IEEE INFOCOM'03, 2003.

[7] Q. Li, J. A. Aslam, and D. Rus, "Online power-aware routing in wireless ad-hoc networks," in Proc. the Seventh Annual International Conference on Mobile Computing and Networking (ACM Mobicom'01), 2001, pp. 97-107.
[8] L. Lin, N. B. Shroff, and R. Srikant, "Energy-aware routing in sensor networks: A large systems approach," in the Third Annual Conference on Wireless On demand Network Systems and Services (WONS), Les Menuires, France, Jan. 2006.

[9] C.-K. Toh, "Maximum battery life routing to support ubiquitous mobile computing in wireless ad hoc networks," IEEE communications Magazine, vol. 39, no. 6, pp. 138-147, June 2001.

[10] Y. Xue, Y. Cui, and K. Nahrstedt, "A utility-based distributed maximum lifetime routing algorithm for wireless networks," in QSHINE, Los Alamitos, CA, USA, 2005.

[11] R. L. Cruz and A. V. Santhanam, "Optimal Routing, Link Scheduling and Power Control in Multi-hop Wireless Networks," in Proceedings of IEEE INFOCOM, San Francisco, April 2003.

[12] M. J. Neely, "Energy Optimal Control for Time Varying Wireless Networks," IEEE Transactions on Information Theory, vol. 52, no. 2 , pp. 2915-2934, July 2006.

[13] X. Lin, N. B. Shroff, and R. Srikant, "A Tutorial on Cross-Layer Optimization in Wireless Networks," IEEE Journal on Selected Areas in Communications, vol. 24, no. 8, pp. 1452-1463, Aug. 2006.

[14] X. Lin and N. B. Shroff, " The Impact of Imperfect Scheduling on CrossLayer Rate Control in Multihop Wireless Networks," in Proceedings of IEEE INFOCOM, Miami, FL, March 2005.

[15] — , "The Impact of Imperfect Scheduling on Cross-Layer Congestion Control in Wireless Networks," IEEE/ACM Transactions on Networking, vol. 14, no. 2, pp. 302-315, 2006.

[16] L. Lin, X. Lin, and N. B. Shroff, "Low-complexity and distributed energy minimization in multi-hop wireless networks," Purdue University, Tech. Rep., 2006, available on http://web.ics.purdue.edu/ 1lin/paper/ tech06.pdf.

[17] S. Boyd and L. Vandenberghe, Convex Optimization. New York, NY, USA: Cambridge University Press, 2004.

[18] X. Lin and N. B. Shroff, "Joint Rate Control and Scheduling in Multihop Wireless Networks," in Proceedings of the IEEE Conference on Decision and Control, Paradise Island, Bahamas, December 2004.

[19] P. Chaporkar, K. Kar, and S. Sarkar, "Throughput Guarantees in Maximal Scheduling in Wireless Networks," in Proceedings of 43d Annual Allerton Conference on Communication, Control and Computing, Monticello, IL, September 2005.

[20] _ - "Achieving Queue Length Stability Through Maximal Scheduling in Wireless Networks," in Proceedings of Information Theory and Applications Inaugural Workshop, University of California, San Diego, February 2006.

[21] X. Wu and R. Srikant, "Bounds on the Capacity Region of Multihop Wireless Networks Under Distributed Greedy Scheduling," in Proceedings of IEEE INFOCOM, Barcelona, Spain, April 2006.

[22] X. Wu, R. Srikant, and J. R. Perkins, "Queue-Length Stability of Maximal Greedy Schedules in Wireless Network," in Proceedings of Information Theory and Applications Inaugural Workshop, University of California, San Diego, February 2006.

[23] B. Hajek and G. Sasaki, "Link Scheduling in Polynomial Time," IEEE Transactions on Information Theory, vol. 34, no. 5, pp. 910-917, September 1988.

[24] D. Bertsekas, A. Nedic, and A. E. Ozdaglar, Convex Analysis and Optimization. Nashua, NH: Athena Scientific, 2003. 